\title{
A Polynomial Time Algorithm to Compute Geodesics in CAT(0) Cubical Complexes
}

\author{
Koyo Hayashi \\ Department of Mathematical Informatics, University of Tokyo, Tokyo 113-8656, Japan \\ koyo_hayashi@mist.i.u-tokyo.ac.jp
}

\begin{abstract}
This paper presents the first polynomial time algorithm to compute geodesics in a CAT(0) cubical complex in general dimension. The algorithm is a simple iterative method to update breakpoints of a path joining two points using Miller, Owen and Provan's algorithm (Adv. in Appl. Math, 2015) as a subroutine. Our algorithm is applicable to any CAT(0) space in which geodesics between two close points can be computed, not limited to CAT(0) cubical complexes.
\end{abstract}

2012 ACM Subject Classification Theory of computation $\rightarrow$ Computational geometry

Keywords and phrases Geodesic, CAT(0) Space, Cubical Complex

Digital Object Identifier 10.4230/LIPIcs.ICALP.2018.78

Related Version A full version of the paper is available at https://arxiv.org/abs/1710. 09932.

Funding The work was supported by JSPS KAKENHI Grant Number 17K00029, and by JST ERATO Grant Number JPMJER1201, Japan.

Acknowledgements I thank Hiroshi Hirai for introducing me to this problem and for helpful comments and careful reading.

\section{Introduction}

Computing a shortest path in a polyhedral domain in Euclidean space is a fundamental and important algorithmic problem, which is intensively studied in computational geometry [16]. This problem is relatively easy to solve in the two-dimensional case; it can generally be reduced to a discrete graph searching problem where some combinatorial approaches can be applied. In three or more dimensions, however, the problem becomes much harder; it is not even discrete. In fact, it was proved by Canny and Reif [8] that the shortest path problem in a polyhedral domain is NP-hard. Mitchell and Sharir [17] have shown that the problem of finding a shortest obstacle-avoiding path is NP-hard even for the case of a region with obstacles that are disjoint axis-aligned boxes. On the other hand, there are some cases where one can obtain polynomial time complexity. For instance, it was shown by Sharir [24] that a shortest obstacle-avoiding path among $k$ disjoint convex polyhedra having altogether $n$ vertices, can be found in $n^{O(k)}$ time, which implies that this problem is polynomially solvable if $k$ is a small constant.

What determines the tractability of the shortest path problem in geometric domains? One of promising answers to this challenging question is global non-positive curvature, or $C A T(0)$ property [14]. CAT(0) spaces are metric spaces in which geodesic triangles are "not thicker" than those in the Euclidean plane, and enjoy various fascinating properties generalizing those in Euclidean and hyperbolic spaces. As Ghrist and LaValle [13] observed, no NP-hard example in [17] is a $\operatorname{CAT}(0)$ space. One of the significant properties of $\operatorname{CAT}(0)$ 
spaces is the uniqueness of geodesics: Every pair of points can be joined by a unique geodesic. Computational and algorithmic theory on $\mathrm{CAT}(0)$ spaces is itself a challenging research field [5].

One of fundamental and familiar CAT(0) spaces is a $C A T(0)$ cubical complex. A cubical complex is a polyhedral complex where each cell is isometric to a unit cube of some dimension and the intersection of any two cells is empty or a single face. Gromov [14] gave a purely combinatorial characterization of cubical complexes of non-positive curvature as cubical complexes in which the link of each vertex is a flag simplicial complex. Chepoi [9] and Roller [22] established that the 1-skeletons of CAT(0) cubical complexes are exactly median graphs, i.e., graphs in which any three vertices admit a unique median vertex. It is also shown by Barthélemy and Constantin [4] that median graphs are exactly the domains of event structures [18]. These nice combinatorial characterizations are one of the main reasons why CAT(0) cubical complexes frequently appear in mathematics, for instance, in geometric group theory [22, 23], metric graph theory [3], concurrency theory in computer science [18], theory of reconfigurable systems $[1,12]$, and phylogenetics [6].

There has been several polynomial time algorithms to find shortest paths in some CAT(0) cubical complexes. A noteworthy example is for a tree space, introduced by Billera, Holmes and Vogtmann [6] as a continuous space of phylogenetic trees. This space is shown to be CAT(0), and consequently provides a powerful tool for comparing two phylogenetic trees through the unique geodesic. Owen and Provan $[19,20]$ gave a polynomial time algorithm for finding geodesics in tree spaces, which was generalized by Miller et al. [15] to CAT(0) orthant spaces, i.e., complexes of Euclidean orthants that are CAT(0). Chepoi and Maftuleac [10] gave an efficient polynomial time algorithm to compute geodesics in a two dimensional CAT $(0)$ cubical complex. These meaningful polynomiality results naturally lead to a question: What about arbitrary CAT(0) cubical complexes?

Ardila, Owen and Sullivant [2] gave a combinatorial description of $\operatorname{CAT}(0)$ cubical complexes, employing a poset endowed with an additional relation, called a poset with inconsistent pairs (PIP). This can be viewed as a generalization of Birkhoff's theorem that gives a compact representation of distributive lattices by posets. In fact, they showed that there is a bijection between CAT(0) cubical complexes and PIPs. (Through the abovementioned equivalence, this can be viewed as a rediscovery of the result of Barthélemy and Constantin [4], who found a bijection between PIPs and pointed median graphs.) This relationship enables us to express an input $\mathrm{CAT}(0)$ cubical complex as a PIP: For a poset with inconsistent pairs $P$, the corresponding $\mathrm{CAT}(0)$ cubical complex $\mathcal{K}_{P}$ is realized as a subcomplex of the $|P|$-dimensional cube $[0,1]^{P}$ in which the cells of $\mathcal{K}_{P}$ are specified by structures of $P$. Adopting this embedding as an input, they gave the first algorithm to compute geodesics in an arbitrary CAT(0) cubical complex. Their algorithm is based on an iterative method to update a sequence of cubes that may contain the geodesic, where at each iteration it solves a touring problem using second order cone programming [21]. They also showed that the touring problem for general CAT(0) cubical complexes has intrinsic algebraic complexity, and geodesics can have breakpoints whose coordinates have nonsolvable Galois group. This implies that there is no exact simple formula for the geodesic and therefore in general, one can only obtain an approximate one. Unfortunately, even if the touring problem could be solved exactly, it is not known whether or not their algorithm is a polynomial one; that is, no polynomial time algorithm has been known for the shortest path problem in a $\mathrm{CAT}(0)$ cubical complex in general dimension. 
Main result. In this paper, we present the first polynomial time algorithm to compute geodesics in a $\mathrm{CAT}(0)$ cubical complex in general dimension, answering the open question suggested by these previous work; namely we show that:

Given a CAT(0) cubical complex $\mathcal{K}$ represented by a poset with inconsistent pairs $P$ and two points $p, q$ in $\mathcal{K}$, one can find a path joining $p$ and $q$ of length at most $d(p, q)+\epsilon$ in time polynomial in $|P|$ and $\log (1 / \epsilon)$.

The algorithm is quite simple, without depending on any involved techniques such as semidefinite programming. To put it briefly, our algorithm first gives a polygonal path joining $p$ and $q$ with a fixed number ( $n$, say) of breakpoints, and then iteratively updates the breakpoints of the path until it becomes a desired one. To update them, we compute the midpoints of the two close breakpoints by using Miller, Owen and Provan's algorithm. The resulting number of iterations is bounded by a polynomial in $n$. Key tools that lead to this bound are linear algebraic techniques and the convexity of the metric of $\operatorname{CAT}(0)$ spaces, rather than inherent properties of cubical complexes. Due to its simplicity, our algorithm is applicable to any CAT(0) space where geodesics between two close points can be found, not limited to $\operatorname{CAT}(0)$ cubical complexes. We believe that our result will be an important step toward developing computational geometry in $\operatorname{CAT}(0)$ spaces.

Application. A reconfigurable system $[1,12]$ is a collection of states which change according to local and reversible moves that affect global positions of the system. Examples include robot motion planning, non-collision particles moving around a graph, and protein folding; see [12]. Abrams, Ghrist and Peterson [1,12] considered a continuous space of all possible positions of a reconfigurable system, called a state complex. Any state complex is a cubical complex of non-positively curved [12], and it becomes CAT(0) in many situations. In the robotics literature, geodesics (in the $l_{2}$-metric) in the $\mathrm{CAT}(0)$ state complex corresponds to the motion planning to get the robot from one position to another one with minimal power consumption. Our algorithm enables us to find such an optimal movement of the robot in polynomial time.

\section{Computing geodesics in CAT(0) spaces}

In this section we devise an algorithm to compute geodesics in general CAT(0) spaces, not limited to $\mathrm{CAT}(0)$ cubical complexes.

\subsection{CAT(0) space}

Let $(X, d)$ be a metric space. A geodesic joining two points $x, y \in X$ is a map $\gamma:[0,1] \rightarrow X$ such that $\gamma(0)=x, \gamma(1)=y$ and $d(\gamma(s), \gamma(t))=d(x, y)|s-t|$ for all $s, t \in[0,1]$. The image of $\gamma$ is called a geodesic segment joining $x$ and $y$. A metric space $X$ is called (uniquely) geodesic if every pair of points $x, y \in X$ is joined by a (unique) geodesic.

For any triple of points $x_{1}, x_{2}, x_{3}$ in a metric space $(X, d)$, there exists a triple of points $\bar{x}_{1}, \bar{x}_{2}, \bar{x}_{3}$ in the Euclidean plane $\mathbb{E}^{2}$ such that $d\left(x_{i}, x_{j}\right)=d_{\mathbb{E}^{2}}\left(\bar{x}_{i}, \bar{x}_{j}\right)$ for $i, j \in\{1,2,3\}$. The Euclidean triangle whose vertices are $\bar{x}_{1}, \bar{x}_{2}$ and $\bar{x}_{3}$ is called a comparison triangle for $x_{1}, x_{2}, x_{3}$. (Note that such a triangle is unique up to isometry.) A geodesic metric space $(X, d)$ is called a $C A T(0)$ space if for any $x_{1}, x_{2}, x_{3} \in X$ and any $p$ belonging to a geodesic segment joining $x_{1}$ and $x_{2}$, the inequality $d\left(x_{3}, p\right) \leq d_{\mathbb{E}^{2}}\left(\bar{x}_{3}, \bar{p}\right)$ holds, where $\bar{p}$ is the unique point in $\mathbb{E}^{2}$ satisfying $d\left(\bar{x}_{i}, \bar{p}\right)=d_{\mathbb{E}^{2}}\left(x_{i}, p\right)$ for $i=1,2$. See Figure 1. 

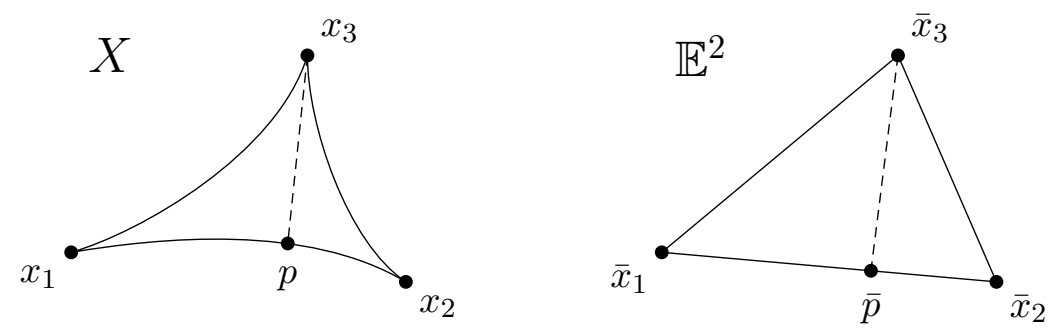

Figure $1 \mathrm{CAT}(0)$ space.

This simple definition yields various significant properties of CAT(0) spaces; see [7] for details. One of the most basic properties of CAT(0) spaces is the convexity of the metric. A geodesic metric space $(X, d)$ is said to be Busemann convex if for any two geodesics $\alpha, \beta:[0,1] \rightarrow X$, the function $f:[0,1] \rightarrow \mathbb{R}$ given by $f(t):=d(\alpha(t), \beta(t))$ is convex.

- Lemma 2.1 ([7, Proposition II.2.2]). Every CAT(0) space is Busemann convex.

A Busemann convex space $X$ is uniquely geodesic. Indeed, for any two geodesics $\alpha, \beta$ : $[0,1] \rightarrow X$ with $\alpha(0)=\beta(0)$ and $\alpha(1)=\beta(1)$, one can easily see that $\alpha$ and $\beta$ coincide, since $d(\alpha(t), \beta(t)) \leq(1-t) d(\alpha(0), \beta(0))+t d(\alpha(1), \beta(1))=0$ for all $t \in[0,1]$. This implies that:

- Theorem 2.2 ([7, Proposition II.1.4]). Every CAT(0) space is uniquely geodesic.

\subsection{Algorithm}

Let $X$ be a CAT(0) space. We shall refer to an element $x$ in the product space $X^{n+1}$ as a chain, and write $x_{i-1}$ to denote the $i$-th component of $x$, i.e., $x=\left(x_{0}, x_{1}, \ldots, x_{n}\right)$. For any chain $x \in X^{n+1}$, we define the length of $x$ by $\sum_{i=0}^{n-1} d\left(x_{i}, x_{i+1}\right)$ and denote it by $\ell(x)$. We consider the following problem:

Given two points $p, q \in X$, a chain $x \in X^{n+1}$ with $x_{0}=p$ and $x_{n}=q$, and a positive parameter $\epsilon>0$, find a chain $y \in X^{n+1}$ such that $y_{0}=p, y_{n}=q$ and

$\ell(y) \leq d(p, q)+\epsilon$,

under the situation where we are given an oracle to perform the following operation for some $D>0$ :

Given two points $p, q \in X$ with $d(p, q) \leq D$, compute the geodesic joining $p$ and $q$ in arbitrary precision.

To explain our algorithm to solve this problem, we need some definitions. Since $X$ is uniquely geodesic, every pair of points $p, q \in X$ has a unique midpoint $w$ satisfying $2 d(w, p)=2 d(q, w)=d(p, q)$. For a nonnegative real number $\delta \geq 0$, a $\delta$-midpoint of $p$ and $q$ is a point $w^{\prime} \in X$ satisfying $d\left(w^{\prime}, w\right) \leq \delta$, where $w$ is the midpoint of $p$ and $q$.

- Definition 2.3 ( $\delta$-halved chain). Let $\delta$ be a nonnegative real number. For any chain $x \in X^{n+1}$, a chain $z \in X^{n+1}$ is called a $\delta$-halved chain of $x$ if it satisfies the following:

$z_{0}=x_{n}, z_{n}=x_{0}$ and $z_{i}$ is a $\delta$-midpoint of $z_{i+1}$ and $x_{n-i}$ for $i=1,2, \ldots, n-1$.

For an integer $k \geq 0$, we say that $x^{(k)}$ is a $k$-th $\delta$-halved chain of $x$ if there exists a sequence $\left\{x^{(j)}\right\}_{j=0}^{k}$ of chains in $X^{n+1}$ such that $x^{(0)}=x$ and $x^{(j)}$ is a $\delta$-halved chain of $x^{(j-1)}$ for $j=1,2, \ldots, k$. 


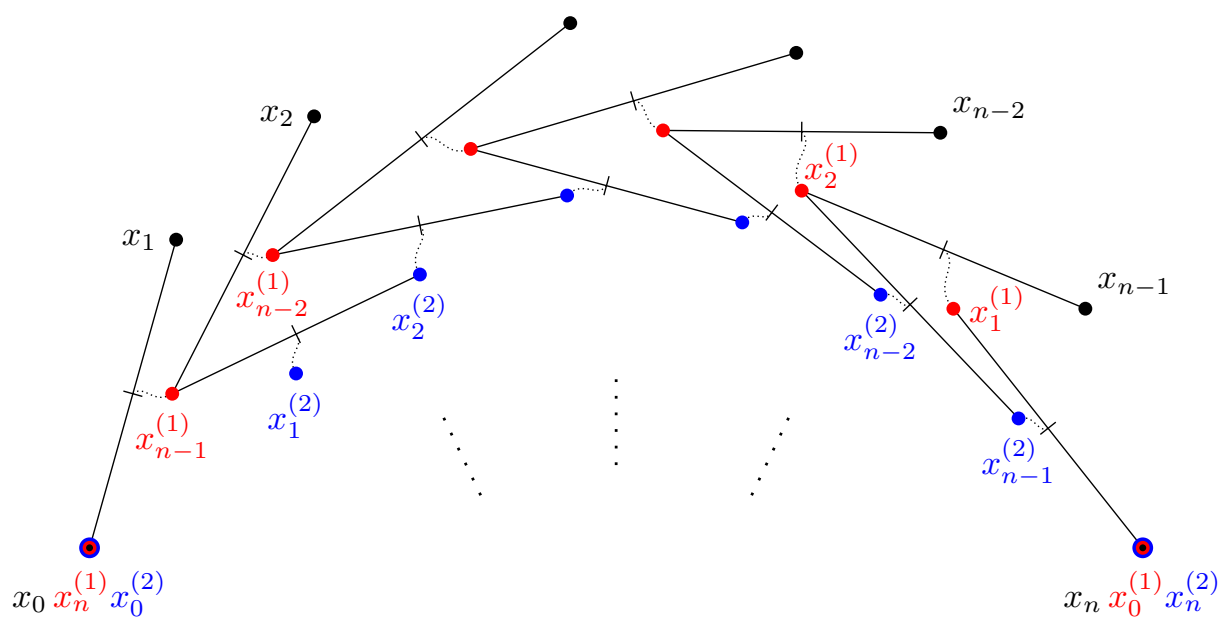

Figure 2 An illustration of Algorithm 1.

Our algorithm can be described as follows. To put it briefly, the algorithm just finds a $k$-th $\delta$-halved chain of a given chain $x$ for some large $k$ and small $\delta$; see Figure 2 for an illustration. In the algorithm the local optimization is done alternatively "from left to right" and "from right to left" so that the analysis will be easier.

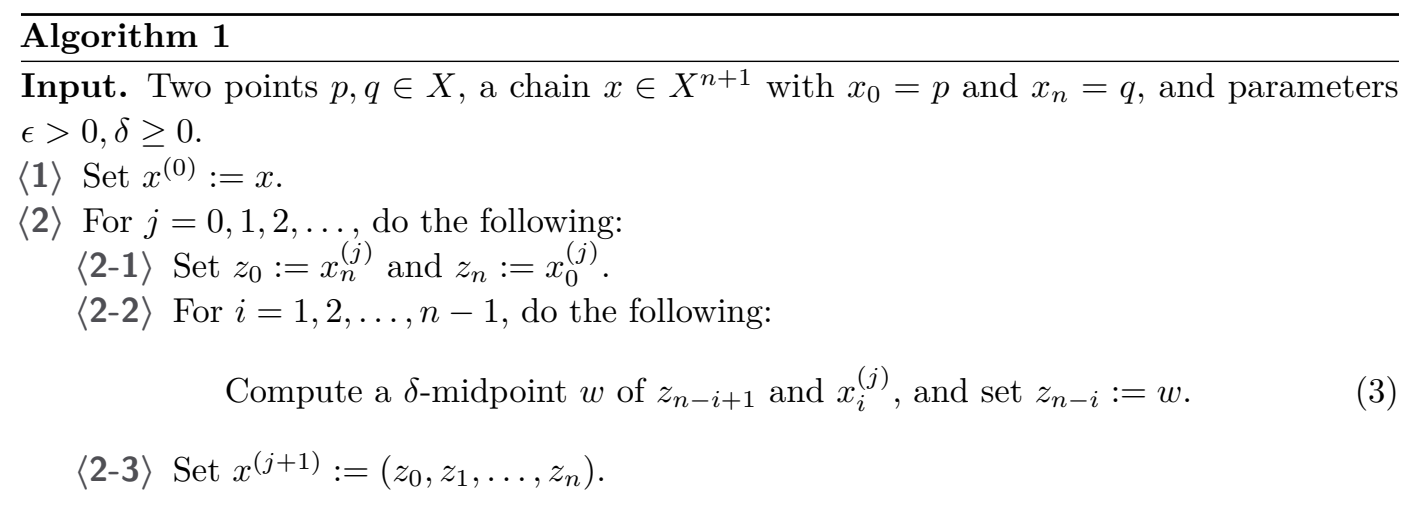

For any chain $x \in X^{n+1}$, define the gap of $x$ by $\max \left\{d\left(x_{0}, x_{1}\right), \max _{1 \leq i \leq n-1} 2 d\left(x_{i}, x_{i+1}\right)\right\}$ and denote it by $\operatorname{gap}(x)$. The following theorem states that Algorithm 1 solves problem (1).

Theorem 2.4. Let $p, q \in X$ be given two points, $x \in X^{n+1}$ be a given chain with $x_{0}=p$ and $x_{n}=q$, and $\epsilon>0,0 \leq \delta \leq \epsilon /\left(16 n^{3}\right)$ be parameters.

(i) For $j \geq n^{2} \log (4 n \cdot \ell(x) / \epsilon)$, one has $\ell\left(x^{(j)}\right) \leq d(p, q)+\epsilon$.

(ii) If $\operatorname{gap}(x) \leq D / 2-\epsilon$ for some $D>0$, then for all $j \geq 0$ and for $i=1,2, \ldots, n-1$, one has $d\left(z_{n-i+1}, x_{i}^{(j)}\right) \leq D$ in (3).

In particular, for $\operatorname{gap}(x) \leq D / 2-\epsilon$, one can find a chain $y \in X^{n+1}$ such that $y_{0}=p, y_{n}=q$ and $\ell(y) \leq d(p, q)+\epsilon$, with $O\left(n^{3} \log (n D / \epsilon)\right)$ calls of an oracle to perform (2).

- Example 2.5. We give an example of CAT(0) spaces to which our algorithm is applicable. A $B_{2}$-complex is a two dimensional piecewise Euclidean complex in which each 2-cell is isomorphic to an isosceles right triangle with short side of length one [11]. A CAT(0) $B_{2^{-}}$ complex is called a folder complex [9]; see Figure 3 for an example. One can show that for a 


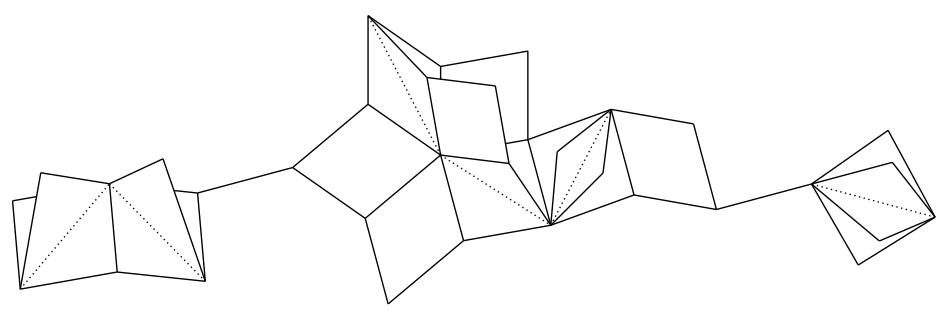

Figure 3 A folder complex.

folder complex $\mathcal{F}$, computing the geodesic between two points $p, q \in \mathcal{F}$ with $d(p, q) \leq 1$ can be reduced to an easy calculation on a subcomplex of $\mathcal{F}$ having a few cells. This implies that our algorithm enables us to find geodesics between two points in a folder complex $\mathcal{F}$ in time bounded by a polynomial in the size of $\mathcal{F}$.

\subsection{Analysis}

For any chain $x \in X^{n+1}$, we define the reference chain $\hat{x} \in X^{n+1}$ of $x$ as follows: $\hat{x}_{0}:=x_{0}$ and $\hat{x}_{i}:=\gamma((i+1) /(n+1))$ for $i=1,2, \ldots, n$, where $\gamma:[0,1] \rightarrow X$ is the geodesic with $\gamma(0)=x_{0}$ and $\gamma(1)=x_{n}$. Reference chains are designed not to be equally spaced but to have a double gap in the beginning so that the analysis of the algorithm will be easier. Note that the reference chain $\hat{x}$ of $x$ is determined just by its end components $x_{0}, x_{n}$, and therefore for any chain $x$ and any even $\delta$-halved chain $x^{(2 k)}$ of $x$ their reference chains coincide: $\hat{x}^{(2 k)}=\hat{x}$. A key observation that leads to Theorem 2.4 is that: For any chain $x \in X^{n+1}$ and any $k$-th $\delta$-halved chain $x^{(k)}$ of $x$ with $k$ sufficiently large and $\delta$ sufficiently small, the distance between $x^{(k)}$ and its reference chain $\hat{x}^{(k)}$ is small enough for its length $\ell\left(x^{(k)}\right)$ to approximate well $d\left(x_{0}, x_{n}\right)$; moreover, the value of such a $k$ can be bounded by a polynomial in $n$. The next lemma states this fact.

- Lemma 2.6. Let $x \in X^{n+1}$. Any $k$-th $\delta$-halved chain $x^{(k)}$ of $x$ satisfies

$$
d\left(x_{i}^{(k)}, \hat{x}_{i}^{(k)}\right) \leq(5 / 4) \ell(x) e^{-k / n^{2}}+3 n^{2} \delta
$$

for $i=1,2, \ldots, n-1$, where $e$ is the base of the natural logarithm.

Proof. Let $\left\{x^{(j)}\right\}_{j \geq 0}$ be a sequence of chains in $X^{n+1}$ such that $x^{(0)}=x$ and $x^{(j)}$ is a $\delta$-halved chain of $x^{(j-1)}$ for $j \geq 1$. Fix an integer $1 \leq i \leq n-1$ and an integer $k \geq 0$. Note that by definition $x_{i}^{(k+1)}$ is a $\delta$-midpoint of $x_{i+1}^{(k+1)}$ and $x_{n-i}^{(k)}$ and that $\hat{x}_{i}^{(k+1)}$ is the midpoint of $\hat{x}_{i+1}^{(k+1)}$ and $\hat{x}_{n-i}^{(k)}$. Hence, by Lemma 2.1 and the triangle inequality, we have

$$
2 d\left(x_{i}^{(k+1)}, \hat{x}_{i}^{(k+1)}\right) \leq 2 d\left(w, \hat{x}_{i}^{(k+1)}\right)+2 \delta \leq d\left(x_{i+1}^{(k+1)}, \hat{x}_{i+1}^{(k+1)}\right)+d\left(x_{n-i}^{(k)}, \hat{x}_{n-i}^{(k)}\right)+2 \delta,
$$

where $w$ is the midpoint of $x_{i+1}^{(k+1)}$ and $x_{n-i}^{(k)}$.

Let $v^{(k)}$ be a column vector of dimension $n-1$ whose $i$-th entry equals $d\left(x_{i}^{(k)}, \hat{x}_{i}^{(k)}\right)$ for $i=1,2, \ldots, n-1$. Let $J$ be a square matrix of order $n-1$ whose $(i, j)$ entry equals 1 if $i+j=n$ and 0 otherwise. Let $K$ be a square matrix of order $n-1$ whose $(i, j)$ entry equals 1 if $j=i+1$ and 0 otherwise. Then, by (4) we have $2 v^{(k+1)} \leq K v^{(k+1)}+J v^{(k)}+2 \delta \mathbf{1}$ for each $k \geq 0$, where 1 is a column vector with all entries equal to 1 . Let $A_{n-1}$ be a square matrix of order $n-1$ whose $(i, j)$ entry equals $(1 / 2)^{n+1-i-j}$ if $i+j \leq n$ and 0 otherwise. Then one can easily see that $(2 I-K)^{-1} J=A_{n-1}$. Hence we have

$$
v^{(k+1)} \leq A_{n-1} v^{(k)}+A_{n-1} J^{-1}(2 \delta \mathbf{1}) \leq A_{n-1} v^{(k)}+2 \delta \mathbf{1}
$$


for each $k \geq 0$. We show that

$$
v^{(k)} \leq\left((5 / 4) \ell(x) e^{-k / n^{2}}+3 n^{2} \delta\right) \mathbf{1}
$$

for any integer $k \geq 0$. The inequality (5) inductively yields that $v^{(k)} \leq\left(A_{n-1}\right)^{k} v^{(0)}+$ $2 \delta\left(I+A_{n-1}+\cdots+\left(A_{n-1}\right)^{k-1}\right) \mathbf{1} \leq \ell(x)\left(A_{n-1}\right)^{k} \mathbf{1}+2 \delta\left(I-A_{n-1}\right)^{-1} \mathbf{1}$. Here, the inequality $v^{(0)} \leq \ell(x) \mathbf{1}$ comes from the triangle inequality. Indeed, we have

$$
\begin{aligned}
d\left(x_{i}, \hat{x}_{i}\right) & \leq \min \left\{d\left(x_{0}, \hat{x}_{i}\right)+\sum_{j=0}^{i-1} d\left(x_{j}, x_{j+1}\right), d\left(\hat{x}_{i}, x_{n}\right)+\sum_{j=i}^{n-1} d\left(x_{j}, x_{j+1}\right)\right\} \\
& \leq\left(d\left(x_{0}, x_{n}\right)+\ell(x)\right) / 2 \leq \ell(x)
\end{aligned}
$$

for $i=1,2, \ldots, n-1$. In Lemma 2.7 below, we prove $\left(I-A_{n-1}\right)^{-1} \mathbf{1} \leq\left(5(n-1)^{2} / 4\right) \mathbf{1}$ (for $n-1 \geq 2)$. This yields that $\left(I-A_{n-1}\right)^{-1} \mathbf{1} \leq(3 / 2) n^{2} \mathbf{1}$ for $n \geq 2$. Also, we prove $\left(A_{n-1}\right)^{k} \mathbf{1} \leq$ $(5 / 4) e^{-k /(n-1)^{2}} \mathbf{1}$ (for $n-1 \geq 2$ ) in Lemma 2.7. This implies that $\left(A_{n-1}\right)^{k} \mathbf{1} \leq(5 / 4) e^{-k / n^{2}} \mathbf{1}$ for $n \geq 2$. This proves (6) and therefore completes the proof of the lemma.

Let us now prove Theorem 2.4.

Proof of Theorem 2.4. We may assume that $n \geq 2$. We first show (i). If $\delta \leq \epsilon /\left(16 n^{3}\right)$ and $j \geq n^{2} \log (4 n \cdot \ell(x) / \epsilon)$, then by Lemma 2.6, any $j$-th $\delta$-halved chain $x^{(j)}$ of $x$ satisfies $d\left(x_{i}^{(j)}, \hat{x}_{i}^{(j)}\right) \leq 5 \epsilon /(16 n)+3 \epsilon /(16 n)=\epsilon /(2 n)$ for $i=1,2, \ldots, n-1$. Hence one has

$$
\begin{aligned}
d\left(x_{i}^{(j)}, x_{i+1}^{(j)}\right) & \leq d\left(x_{i}^{(j)}, \hat{x}_{i}^{(j)}\right)+d\left(\hat{x}_{i}^{(j)}, \hat{x}_{i+1}^{(j)}\right)+d\left(\hat{x}_{i+1}^{(j)}, x_{i+1}^{(j)}\right) \\
& \leq d\left(\hat{x}_{i}^{(j)}, \hat{x}_{i+1}^{(j)}\right)+\epsilon / n
\end{aligned}
$$

for $i=0,1, \ldots, n-1$. This implies that $\ell\left(x^{(j)}\right)=\sum_{i=0}^{n-1} d\left(x_{i}^{(j)}, x_{i+1}^{(j)}\right) \leq \sum_{i=0}^{n-1}\left(d\left(\hat{x}_{i}^{(j)}, \hat{x}_{i+1}^{(j)}\right)+\right.$ $\epsilon / n)=d\left(x_{0}, x_{n}\right)+\epsilon=d(p, q)+\epsilon$, and therefore completes the proof of (i).

To prove (ii), we first show

$$
d\left(z_{n-i+1}, x_{i}^{(j)}\right) \leq \operatorname{gap}\left(x^{(j)}\right)+2 \delta \quad(i=1,2, \ldots, n ; j \geq 0),
$$

by induction on $i$. The case $i=1$ being trivial, suppose that $i \geq 2$. Since $z_{n-i+1}$ is a $\delta$ midpoint of $z_{n-i+2}$ and $x_{i-1}^{(j)}$, the triangle inequality and the induction yield $d\left(z_{n-i+1}, x_{i}^{(j)}\right) \leq$ $\delta+d\left(z_{n-i+2}, x_{i-1}^{(j)}\right) / 2+d\left(x_{i-1}^{(j)}, x_{i}^{(j)}\right) \leq \delta+\left(\operatorname{gap}\left(x^{(j)}\right) / 2+\delta\right)+\operatorname{gap}\left(x^{(j)}\right) / 2=\operatorname{gap}\left(x^{(j)}\right)+2 \delta$, which completes the induction.

It follows from (8) that $\operatorname{gap}\left(x^{(j+1)}\right) \leq \operatorname{gap}\left(x^{(j)}\right)+4 \delta$ for $j \geq 0$. Indeed, the case $i=n$ in (8) implies that $d\left(z_{1}, z_{0}\right)=d\left(z_{1}, x_{n}^{(j)}\right) \leq \operatorname{gap}\left(x^{(j)}\right)+2 \delta$; on the other hand, by the triangle inequality and (8), one has $d\left(z_{n-i+1}, z_{n-i}\right) \leq d\left(z_{n-i+1}, x_{i}^{(j)}\right) / 2+\delta \leq \operatorname{gap}\left(x^{(j)}\right) / 2+2 \delta$ for $i=1,2, \ldots, n-1$. Thus, one has $\operatorname{gap}\left(x^{(j+1)}\right) \leq \max \left\{\operatorname{gap}\left(x^{(j)}\right)+2 \delta, 2\left(\operatorname{gap}\left(x^{(j)}\right) / 2+2 \delta\right)\right\}=$ $\operatorname{gap}\left(x^{(j)}\right)+4 \delta$.

The inequality (8) implies that in order to prove (ii) it suffices to show that $\operatorname{gap}\left(x^{(j)}\right)+2 \delta \leq$ $D$ for all $j \geq 0$. Suppose that $\delta \leq \epsilon /\left(16 n^{3}\right)$. We consider two cases.

Case 1: $j \leq n^{2} \log (4 n \cdot \ell(x) / \epsilon)$. Note that $\ell(x) \leq n \cdot \operatorname{gap}(x)$ and that $\operatorname{gap}\left(x^{(j)}\right) \leq \operatorname{gap}(x)+4 j \delta$.

However roughly one estimates an upper bound of $4 j \delta$, one can get

$$
4 j \delta \leq 4 \cdot \frac{\epsilon}{16 n^{3}} \cdot n^{2} \log \frac{4 n^{2} \cdot \operatorname{gap}(x)}{\epsilon}=\frac{\epsilon}{4 n}\left(\log \frac{\operatorname{gap}(x)}{\epsilon}+2 \log 2 n\right) \leq \frac{\operatorname{gap}(x)}{4 n e}+\frac{\epsilon}{e},
$$

where the last inequality comes from the fact that $\log t \leq t / e$ for any $t>0$. It is easy to see that $\operatorname{gap}\left(x^{(j)}\right)+2 \delta \leq \operatorname{gap}(x)+\operatorname{gap}(x) /(4 n e)+\epsilon / e+\epsilon /\left(8 n^{3}\right) \leq D$, provided $\operatorname{gap}(x) \leq D / 2-\epsilon$. 
Case 2: $j \geq n^{2} \log (4 n \cdot \ell(x) / \epsilon)$. Recall (7). Since $d\left(x_{0}, x_{n}\right) /(n+1) \leq \operatorname{gap}(x) / 2$, we have

$$
\operatorname{gap}\left(x^{(j)}\right) \leq \max \{\operatorname{gap}(x)+\epsilon / n, 2(\operatorname{gap}(x) / 2+\epsilon / n)\}=\operatorname{gap}(x)+2 \epsilon / n .
$$

It is easy to see that $\operatorname{gap}\left(x^{(j)}\right)+2 \delta \leq \operatorname{gap}(x)+2 \epsilon / n+\epsilon /\left(8 n^{3}\right) \leq D$, provided $\operatorname{gap}(x) \leq$ $D / 2-\epsilon$.

From (i) and (ii), we can show the last part of the theorem. Indeed, for $k:=\left\lceil n^{2} \log (4 n\right.$. $\ell(x) / \epsilon)\rceil$, one can find a $k$-th $\delta$-halved chain $x^{(k)}$ of $x$ with $O(n k)=O\left(n^{3} \log (n D / \epsilon)\right)$ oracle calls, from (ii); its length $\ell\left(x^{(k)}\right)$ is at most $d(p, q)+\epsilon$, from (i).

We end this section by showing the lemma used in the proof of Lemma 2.6. Let $A_{n}$ be an $n \times n$ matrix whose $(i, j)$ entry is defined by

$$
\left(A_{n}\right)_{i j}:= \begin{cases}(1 / 2)^{n+2-i-j} & (i+j \leq n+1) \\ 0 & \text { (otherwise) }\end{cases}
$$

for $i, j=1,2, \ldots, n$. Since $A_{n}$ is a nonnegative matrix, its spectral radius $\rho\left(A_{n}\right)$ is at most the maximum row sum of $A_{n}$, which immediately yields that $\rho\left(A_{n}\right) \leq 1-(1 / 2)^{n}$. This inequality, however, is not tight unless $n=1$. In fact, one can obtain a more useful upper bound of $\rho\left(A_{n}\right)$.

- Lemma 2.7. Let $n>1$ be an integer, and let $A_{n}$ be an $n \times n$ matrix defined by (9). Then its spectral radius $\rho\left(A_{n}\right)$ is at most $1-1 / n^{2}$. In addition, one has $\left(I-A_{n}\right)^{-1} \mathbf{1} \leq\left(5 n^{2} / 4\right) \mathbf{1}$ and $\left(A_{n}\right)^{k} \mathbf{1} \leq(5 / 4) e^{-k / n^{2}} \mathbf{1}$ for any integer $k \geq 0$.

Proof. Let $A:=A_{n}$ for simplicity. Let $u$ be a positive column vector of dimension $n$ whose $k$-th entry is defined by $u_{k}:=k(n-k)+n^{2}$ for $k=1,2, \ldots, n$. By the Collatz-Wielandt inequality, in order to show $\rho(A) \leq 1-1 / n^{2}$ it suffices to show that $A u \leq\left(1-1 / n^{2}\right) u$. The $k$-th entry of the vector $A u$ is

$$
(A u)_{k}=\sum_{j=1}^{n+1-k} \frac{u_{j}}{2^{n+2-k-j}}=\frac{1}{2^{n+2-k}} \sum_{j=1}^{n+1-k} 2^{j}\left(-j^{2}+n j+n^{2}\right) .
$$

Hence, using the general formulas

$$
\sum_{j=1}^{m} j \cdot 2^{j}=2+2^{m+1}(m-1) \quad \text { and } \quad \sum_{j=1}^{m} j^{2} \cdot 2^{j}=-6+2^{m+1}\left((m-1)^{2}+2\right),
$$

we have

$$
(A u)_{k}=u_{k}-2-\frac{n^{2}-n-3}{2^{n+1-k}} .
$$

It is easy to see that for $n \geq 2$ and $1 \leq k \leq n$ one has

$$
\frac{u_{k}}{n^{2}}=1+\frac{k(n-k)}{n^{2}} \leq \frac{5}{4} \leq\left(2-\frac{1}{2^{n+1-k}}\right)+\frac{(n-2)(n+1)}{2^{n+1-k}},
$$

which implies that

$$
\frac{u_{k}}{n^{2}} \leq 2+\frac{n^{2}-n-3}{2^{n+1-k}} \quad(k=1,2, \ldots, n) .
$$

This completes the proof of the inequality $A u \leq\left(1-1 / n^{2}\right) u$. 
Let us show the latter part of the lemma. Note that $1 \leq\left(1 / n^{2}\right) u \leq(5 / 4) \mathbf{1}$. Since $\left(1 / n^{2}\right) u \leq(I-A) u$ and $(I-A)^{-1}$ is a nonnegative matrix (as $\left.\rho(A)<1\right)$, we have $(I-A)^{-1} \mathbf{1} \leq$ $\left(1 / n^{2}\right)(I-A)^{-1} u \leq u \leq\left(5 n^{2} / 4\right) \mathbf{1}$.

Since $A u \leq\left(1-1 / n^{2}\right) u \leq e^{-1 / n^{2}} u$, we have $A^{k} u \leq e^{-k / n^{2}} u$ for any integer $k \geq 0$. Hence, $A^{k} \mathbf{1} \leq\left(1 / n^{2}\right) A^{k} u \leq\left(1 / n^{2}\right) e^{-k / n^{2}} u \leq(5 / 4) e^{-k / n^{2}} \mathbf{1}$.

- Remark. In proving Theorem 2.4, we utilized only the convexity of the metric of $X$. Hence our algorithm works even when $X$ is a Busemann convex space.

\section{Computing geodesics in CAT(0) cubical complexes}

In this section we give an algorithm to compute geodesics in CAT(0) cubical complexes, with an aid of the result of the preceding section. In Section 3.1 to 3.4, we recall $\operatorname{CAT}(0)$ cubical complexes, median graphs, PIPs and CAT(0) orthant spaces. Section 3.5 is devoted to proving our main theorem.

\subsection{CAT(0) cubical complex}

A cubical complex $\mathcal{K}$ is a polyhedral complex where each $k$-dimensional cell is isometric to the unit cube $[0,1]^{k}$ and the intersection of any two cells is empty or a single face. The underlying graph of $\mathcal{K}$ is the graph $G(\mathcal{K})=(V(\mathcal{K}), E(\mathcal{K}))$, where $V(\mathcal{K})$ denotes the set of vertices (0-dimensional faces) of $\mathcal{K}$ and $E(\mathcal{K})$ denotes the set of edges (1-dimensional faces) of $\mathcal{K}$. A cubical complex $\mathcal{K}$ has an intrinsic metric induced by the $l_{2}$-metric on each cell. For two points $p, q \in \mathcal{K}$, a string in $\mathcal{K}$ from $p$ to $q$ is a sequence of points $p=x_{0}, x_{1}, \ldots, x_{m-1}, x_{m}=q$ in $\mathcal{K}$ such that for each $i=0,1, \ldots, m-1$ there exists a cell $C_{i}$ containing $x_{i}$ and $x_{i+1}$, and its length is defined to be $\sum_{i=0}^{m-1} d\left(x_{i}, x_{i+1}\right)$, where $d\left(x_{i}, x_{i+1}\right)$ is measured inside $C_{i}$ by the $l_{2}$-metric. The distance between two points $p, q \in \mathcal{K}$ is defined to be the infimum of the lengths of strings from $p$ to $q$.

Gromov [14] gave a combinatorial criterion which allows us to easily decide whether or not a cubical complex $\mathcal{K}$ is non-positively curved. The link of a vertex $v$ of $\mathcal{K}$ is the abstract simplicial complex whose vertices are the edges of $\mathcal{K}$ containing $v$ and where $k$ edges $e_{1}, \ldots, e_{k}$ span a simplex if and only if they are contained in a common $k$-dimensional cell of $\mathcal{K}$. An abstract simplicial complex $\mathcal{L}$ is called flag if any set of vertices is a simplex of $\mathcal{L}$ whenever each pair of its vertices spans a simplex.

- Theorem 3.1 (Gromov [14]). A cubical complex $\mathcal{K}$ is $C A T(0)$ if and only if $\mathcal{K}$ is simply connected and the link of each vertex is flag.

\subsection{Median graph}

Let $G=(V, E)$ be a simple undirected graph. The distance $d_{G}(u, v)$ between two vertices $u$ and $v$ is the length of a shortest path between $u$ and $v$. The interval $I_{G}(u, v)$ between $u$ and $v$ is the set of vertices $w \in V$ with $d_{G}(u, v)=d_{G}(u, w)+d_{G}(w, v)$. A vertex subset $U \subseteq V$ is said to be convex if $I_{G}(u, v)$ is contained in $U$ for all $u, v \in U$. A graph $G$ is called a median graph if for all $u, v, w \in V$ the set $I_{G}(u, v) \cap I_{G}(v, w) \cap I_{G}(w, u)$ contains exactly one element, called the median of $u, v, w$. Median graphs are connected and bipartite. A median complex is a cubical complex derived from a median graph $G$ by replacing all cube-subgraphs of $G$ by solid cubes. It has been shown independently by Chepoi [9] and Roller [22] that median complexes and $\mathrm{CAT}(0)$ cubical complexes constitute the same objects: 

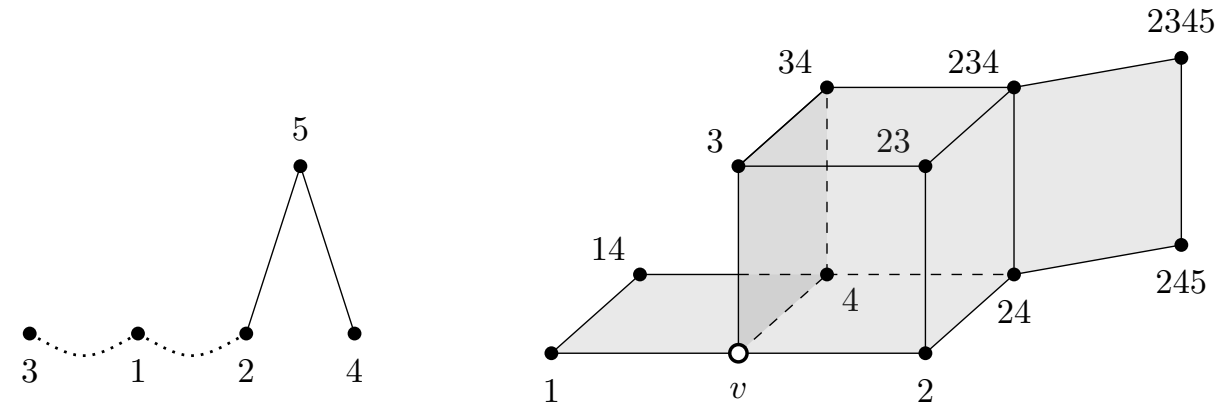

Figure 4 A poset with inconsistent pairs and the corresponding rooted CAT(0) cubical complex. Dotted line represents minimal inconsistent pairs, where an inconsistent pair $\{a, b\}$ is said to be minimal if there is no other inconsistent pair $\left\{a^{\prime}, b^{\prime}\right\}$ with $a^{\prime} \preceq a$ and $b^{\prime} \preceq b$.

- Theorem 3.2 (Chepoi [9], Roller [22]). The underlying graph of every CAT(0) cubical complex is a median graph, and conversely, every median complex is a CAT(0) cubical complex.

\subsection{Poset with inconsistent pairs (PIP)}

Barthélemy and Constantin [4] established a Birkhoff-type representation theorem for median graphs, by employing a poset with an additional relation. This structure was rediscovered by Ardila et al. [2] in the context of CAT(0) cubical complexes. An antichain of a poset $P$ is a subset of $P$ that contains no two comparable elements. A subset $I$ of $P$ is called an order ideal of $P$ if $a \in I$ and $b \preceq a$ imply $b \in I$. A poset $P$ is locally finite if every interval $[a, b]=\{c \in P \mid a \preceq c \preceq b\}$ is finite, and it has finite width if every antichain is finite.

- Definition 3.3. A poset with inconsistent pairs (or, briefly, a PIP) is a locally finite poset $P$ of finite width, endowed with a symmetric binary relation $\smile$ satisfying:

1) If $a \smile b$, then $a$ and $b$ are incomparable.

2) If $a \smile b, a \preceq a^{\prime}$ and $b \preceq b^{\prime}$, then $a^{\prime} \smile b^{\prime}$.

A pair $\{a, b\}$ with $a \smile b$ is called an inconsistent pair. An order ideal of $P$ is called consistent if it contains no inconsistent pairs.

For a $\mathrm{CAT}(0)$ cubical complex $\mathcal{K}$ and a vertex $v$ of $\mathcal{K}$, the pair $(\mathcal{K}, v)$ is called a rooted $C A T(0)$ cubical complex. Given a poset with inconsistent pairs $P$, one can construct a cubical complex $\mathcal{K}_{P}$ as follows: The underlying graph $G\left(\mathcal{K}_{P}\right)$ is a graph $G_{P}$ whose vertices are consistent order ideals of $P$ and where two consistent order ideals $I, J$ are adjacent if and only if $|I \Delta J|=1$; replace all cube-subgraphs (i.e., subgraphs isomorphic to cubes of some dimensions) of $G_{P}$ by solid cubes. See Figure 4 for an example. In fact, the resulting cubical complex $\mathcal{K}_{P}$ is $\operatorname{CAT}(0)$, and moreover:

Theorem 3.4 (Ardila et al. [2]). The map $P \mapsto \mathcal{K}_{P}$ is a bijection between posets with inconsistent pairs and rooted $C A T(0)$ cubical complexes.

This bijection can also be derived from Theorem 3.2 and the result of Barthélemy and Constantin [4], who found a bijection between PIPs and pointed median graphs.

Given a poset with inconsistent pairs $P$, one can embed $\mathcal{K}_{P}$ into a unit cube in the Euclidean space as follows, which we call the standard embedding of $P[2]$ :

$$
\mathcal{K}_{P}=\left\{\left(x_{i}\right)_{i \in P} \in[0,1]^{P} \mid i \prec j \text { and } x_{i}<1 \Rightarrow x_{j}=0 \text {, and } i \smile j \Rightarrow x_{i} x_{j}=0\right\} .
$$


For each pair $(I, M)$ of a consistent order ideal $I$ of $P$ and a subset $M \subseteq I_{\max }$, where $I_{\max }$ is the set of maximal elements of $I$, the subspace

$$
C_{M}^{I}:=\left\{x \in \mathcal{K}_{P} \mid i \in I \backslash M \Rightarrow x_{i}=1 \text {, and } i \notin I \Rightarrow x_{i}=0\right\}=\{1\}^{I \backslash M} \times[0,1]^{M} \times\{0\}^{P \backslash I}
$$

corresponds to a unique $|M|$-dimensional cell of $\mathcal{K}_{P}$.

\subsection{CAT(0) orthant space}

Let $\mathbb{R}_{+}$denote the set of nonnegative real numbers. Let $\mathcal{L}$ be an abstract simplicial complex on a finite set $V$. The orthant space $\mathcal{O}(\mathcal{L})$ for $\mathcal{L}$ is a subspace of $|V|$-dimensional orthant $\mathbb{R}_{+}^{V}$ constructed by taking a union of all subcones $\left\{\mathcal{O}_{S} \mid S \in \mathcal{L}\right\}$ associated with simplices of $\mathcal{L}$, where $\mathcal{O}_{S}$ is defined by $\mathcal{O}_{S}:=\mathbb{R}_{+}^{S} \times\{0\}^{V \backslash S}$ for each simplex $S \in \mathcal{L}$; namely, $\mathcal{O}(\mathcal{L})=\bigcup_{S \in \mathcal{L}}\left\{x \in \mathbb{R}_{+}^{V} \mid x_{v}=0\right.$ for each $\left.v \notin S\right\}$. The distance between two points $x, y \in \mathcal{O}(\mathcal{L})$ is defined in a similar way as in the case of cubical complexes. An orthant space is a special instance of cubical complexes.

- Theorem 3.5 (Gromov [14]). The orthant space $\mathcal{O}(\mathcal{L})$ for an abstract simplicial complex $\mathcal{L}$ is a $C A T(0)$ space if and only if $\mathcal{L}$ is a flag complex.

A typical example of $\operatorname{CAT}(0)$ orthant spaces is a tree space [6]. Owen and Provan [19, 20] gave a polynomial time algorithm to compute geodesics in tree spaces, which was generalized to $\mathrm{CAT}(0)$ orthant spaces by Miller et al. [15].

- Theorem $3.6([15,19,20])$. Let $\mathcal{L}$ be a flag abstract simplicial complex on a finite set $V$ and $\mathcal{O}(\mathcal{L})$ be the $C A T(0)$ orthant space for $\mathcal{L}$. Let $x, y \in \mathcal{O}(\mathcal{L})$, and let $S_{1}$ and $S_{2}$ be the inclusion-wise minimal simplices such that $x \in \mathcal{O}_{S_{1}}$ and $y \in \mathcal{O}_{S_{2}}$. Then one can find the explicit description of the geodesic joining $x$ and $y$ in $O\left(\left(\left|S_{1}\right|+\left|S_{2}\right|\right)^{4}\right)$ time.

An interesting thing about their algorithm is that it solves as a subproblem a combinatorial optimization problem: the Maximum Weight Stable Set problem on a bipartite graph whose color classes have at most $\left|S_{1}\right|,\left|S_{2}\right|$ vertices, respectively. We should note that the above explicit descriptions of geodesics are radical expressions. Computationally, for a point $p$ on a geodesic, one can compute a rational point $p^{\prime} \in \mathcal{O}(\mathcal{L})$ such that $d\left(p^{\prime}, p\right) \leq \delta$ and the number of bits required for each coordinate of $p^{\prime}$ is bounded by $O(\log (|V| / \delta))$. For a real number $r>0$, the subspace $\mathcal{O}(\mathcal{L}) \cap[0, r]^{V}$ of $\mathcal{O}(\mathcal{L})$, denoted by $\left.\mathcal{O}(\mathcal{L})\right|_{[0, r]}$, is called a truncated $C A T(0)$ orthant space. Actually, from the explicit descriptions of geodesics in $\mathcal{O}(\mathcal{L})$, one can see that $\left.\mathcal{O}(\mathcal{L})\right|_{[0, r]}$ is convex in $\mathcal{O}(\mathcal{L})$, and thus:

Theorem 3.7 ([15]). Given two points $x, y$ in a truncated $C A T(0)$ orthant space $\left.\mathcal{O}(\mathcal{L})\right|_{[0, r]}$, one can find the explicit description of the geodesic joining $x$ and $y$ in $O\left(|V|^{4}\right)$ time.

\subsection{Main theorem}

Our main result is the following theorem. It should be remarked that as stated in [2] there are no simple formulas for the breakpoints in geodesics in $\mathrm{CAT}(0)$ cubical complexes due to their algebraic complexity, and hence one can only compute them approximately. Also note that for the shortest path problem in a general CAT(0) cubical complex there has been no algorithm that runs in time polynomial in the size of the complex, much less the size of the compact representation PIP. 
- Problem 3.8. Given a poset with inconsistent pairs $P$, two points $p, q$ in the standard embedding $\mathcal{K}_{P}$ of $P$, and a positive parameter $\epsilon>0$, find a sequence of points $p=$ $x_{0}, x_{1}, \ldots, x_{n-1}, x_{n}=q$ in $\mathcal{K}_{P}$ with $\sum_{i=0}^{n-1} d\left(x_{i}, x_{i+1}\right) \leq d(p, q)+\epsilon$ and compute the geodesic joining $x_{i}$ and $x_{i+1}$ for $i=0,1, \ldots, n-1$.

- Theorem 3.9. Problem 3.8 can be solved in $O\left(|P|^{7} \log (|P| / \epsilon)\right)$ time. Moreover, the number of bits required for each coordinate of points in $\mathcal{K}_{P}$ occurring throughout the algorithm can be bounded by $O(\log (|P| / \epsilon))$.

Let us show this theorem. Let $m$ denote the number of elements of $P$ and let $D<1$ be a positive constant close to 1 (e.g., set $D:=0.9$ ). Theorem 2.4 implies that in order to prove Theorem 3.9 it suffices to show that:

(a) Given two points $p, q \in \mathcal{K}_{P}$, one can find a sequence of points $p=x_{0}, x_{1}, \ldots, x_{n-1}, x_{n}=q$ in $\mathcal{K}_{P}$ such that $n=O(m)$ and $d\left(x_{i}, x_{i+1}\right) \leq D / 4-\epsilon$ for $i=0,1, \ldots, n-1$.

(b) Given two points $p, q \in \mathcal{K}_{P}$ with $d(p, q) \leq D$, one can compute the geodesic joining $p$ and $q$ in $O\left(m^{4}\right)$ time and find a $\delta$-midpoint $w$ of $p$ and $q$ with $O(\log (m / \delta))$ bits enough for each coordinate of $w$.

It is relatively easy to show (a), by considering a curve $c(p, q)$ issuing at $p$, going through an edge geodesic (a shortest path in the underlying graph of $\mathcal{K}_{P}$ ) between some vertices of cells containing $p, q$, and ending at $q$. (Note that one can easily find an edge geodesic between vertices $u$ and $v$ of $\mathcal{K}_{P}$. Reroot the complex $\mathcal{K}_{P}$ at $u$. In other words, construct a poset $P^{\prime}$ for which $\mathcal{K}_{P^{\prime}} \cong \mathcal{K}_{P}$ and $u$ is the root of $\mathcal{K}_{P^{\prime}}$; this construction is implicitly stated in [2]. Then the edge geodesic in $\mathcal{K}_{P^{\prime}}$ from the root $u=\emptyset$ to $v=I$, where $I$ is a consistent order ideal of $P^{\prime}$, can be found by considering a linear extension of the elements of $I$.) Since such a curve $c(p, q)$ has length at most $O(m)$, dividing it into parts appropriately, one can get a desired sequence of points. To show (b), we need the following two lemmas, whose detailed proofs can be found in the full version of this work.

- Lemma 3.10. Let $\mathcal{K}$ be a $C A T(0)$ cubical complex and $v$ be a vertex of $\mathcal{K}$. Then the star $\operatorname{St}(v, \mathcal{K})$ of $v$ in $\mathcal{K}$, i.e., the subcomplex spanned by all cells containing $v$, is convex in $\mathcal{K}$.

Sketch of Proof. The lemma follows from the well-known fact that the vertex set of a star in $\mathcal{K}$ is convex in the underlying graph $G(\mathcal{K})$ and the result of [10] that the subcomplex $\mathcal{K}(S)$ of $\mathcal{K}$ induced by a convex vertex subset $S$ of $G(\mathcal{K})$ is convex in $\mathcal{K}$ in the $\ell_{2}$-metric.

- Lemma 3.11. Let $\mathcal{K}$ be a $C A T(0)$ cubical complex. Let $p, q$ be two points in $\mathcal{K}$ with $d(p, q)<$ 1 and $R_{1}, R_{2}$ be the minimal cells of $\mathcal{K}$ containing $p, q$, respectively. Then $R_{1} \cap R_{2} \neq \emptyset$.

Sketch of Proof. One can show that there exists a vertex $u_{i}$ of $R_{i}$ for $i=1,2$, such that $d\left(u_{1}, u_{2}\right)=d\left(R_{1}, R_{2}\right):=\inf _{x \in R_{1}, y \in R_{2}} d(x, y)$. Since $d\left(R_{1}, R_{2}\right) \leq d(p, q)<1$, one has $d\left(u_{1}, u_{2}\right)<1$. Hence $u_{1}$ and $u_{2}$ should be the same vertex, and thus $R_{1} \cap R_{2} \neq \emptyset$.

Using these lemmas, we show (b). Suppose that we are given two points $p, q \in \mathcal{K}_{P}$ with $d(p, q) \leq D$. First notice that one can find in linear time the minimal cells $R_{1}$ and $R_{2}$ of $\mathcal{K}_{P}$ that contain $p$ and $q$, respectively, just by checking their coordinates. (Indeed, one has $R_{1}=C_{M}^{I}$ for $I=\left\{i \in P \mid p_{i}>0\right\}$ and $M=\left\{i \in P \mid 0<p_{i}<1\right\}$.) Since $d(p, q) \leq D<1$, from Lemma 3.11 we know that $R_{1} \cap R_{2} \neq \emptyset$. Let $v$ be a vertex of $R_{1} \cap R_{2}$. Then $p$ and $q$ are contained in the $\operatorname{star} \operatorname{St}\left(v, \mathcal{K}_{P}\right)$ of $v$. Since $\operatorname{St}\left(v, \mathcal{K}_{P}\right)$ is convex in $\mathcal{K}_{P}$ by Lemma 3.10, we only have to compute the geodesic in $\operatorname{St}\left(v, \mathcal{K}_{P}\right)$. Obviously, $\operatorname{St}\left(v, \mathcal{K}_{P}\right)$ is a truncated $\operatorname{CAT}(0)$ orthant space, and hence one can compute the geodesic between $p$ and $q$ in $\operatorname{St}\left(v, \mathcal{K}_{P}\right)$ in $O\left(m^{4}\right)$ time, by Theorem 3.7. In addition, one can find a $\delta$-midpoint $w \in \operatorname{St}\left(v, \mathcal{K}_{P}\right)$ of $p$ and $q$ such that the number of bits required for each coordinate of $w$ is bounded by $O(\log (m / \delta))$. This implies (b) and therefore completes the proof of Theorem 3.9. 


\section{References}

1 Aaron Abrams and Robert Ghrist. State complexes for metamorphic robots. The International Journal of Robotics Research, 23:811-826, 2004.

2 Federico Ardila, Megan Owen, and Seth Sullivant. Geodesics in CAT(0) cubical complexes. Adv. in Appl. Math., 48:142-163, 2012.

3 Hans-Jürgen Bandelt and Victor Chepoi. Metric graph theory and geometry: a survey. In Jacob E. Goodman, János Pach, and Richard Pollack, editors, Surveys on discrete and computational geometry, volume 453 of Contemp. Math., pages 49-86. Amer. Math. Soc., Providence, RI, 2008.

4 Jean-Pierre Barthélemy and Julien Constantin. Median graphs, parallelism and posets. Discrete Math., 111:49-63, 1993.

5 Miroslav Bačák. Convex analysis and optimization in Hadamard spaces. De Gruyter, Berlin, 2014.

6 Louis J. Billera, Susan P. Holmes, and Karen Vogtmann. Geometry of the space of phylogenetic trees. Adv. in Appl. Math., 27:733-767, 2001.

7 Martin R. Bridson and André Haefliger. Metric spaces of non-positive curvature. SpringerVerlag, Berlin, 1999.

8 John Canny and John Reif. New lower bound techniques for robot motion planning problems. In Proceedings of the 28th Annual Symposium on Foundations of Computer Science, pages 49-60, 1987.

9 Victor Chepoi. Graphs of some CAT(0) complexes. Adv. in Appl. Math., 24:125-179, 2000.

10 Victor Chepoi and Daniela Maftuleac. Shortest path problem in rectangular complexes of global nonpositive curvature. Comput. Geom., 46:51-64, 2013.

11 S. M. Gersten and H. Short. Small cancellation theory and automatic groups. II. Invent. Math., 105:641-662, 1991.

12 R. Ghrist and V. Peterson. The geometry and topology of reconfiguration. Adv. in Appl. Math., 38:302-323, 2007.

13 Robert Ghrist and Steven M. LaValle. Nonpositive curvature and Pareto optimal coordination of robots. SIAM J. Control Optim., 45:1697-1713, 2006.

14 M. Gromov. Hyperbolic groups. In S. M. Gersten, editor, Essays in group theory, volume 8 of Math. Sci. Res. Inst. Publ., pages 75-263. Springer, New York, 1987.

15 Ezra Miller, Megan Owen, and J. Scott Provan. Polyhedral computational geometry for averaging metric phylogenetic trees. Adv. in Appl. Math., 68:51-91, 2015.

16 Joseph S. B. Mitchell. Geometric shortest paths and network optimization. In J.-R. Sack and J. Urrutia, editors, Handbook of computational geometry, pages 633-701. NorthHolland, Amsterdam, 2000.

17 Joseph S. B. Mitchell and Micha Sharir. New results on shortest paths in three dimensions. In Proceedings of the 20th Annual Symposium on Computational Geometry, pages 124-133, 2004.

18 Mogens Nielsen, Gordon Plotkin, and Glynn Winskel. Petri nets, event structures and domains. I. Theoret. Comput. Sci., 13:85-108, 1981.

19 Megan Owen. Computing geodesic distances in tree space. SIAM J. Discrete Math., 25:1506-1529, 2011.

20 Megan Owen and J. Scott Provan. A fast algorithm for computing geodesic distances in tree space. IEEE/ACM Transactions on Computational Biology and Bioinformatics, 8:2-13, 2011.

21 Valentin Polishchuk and Joseph S. B. Mitchell. Touring convex bodies - A conic programming solution. In Proceedings of the 17th Canadian Conference on Computational Geometry, pages 101-104, 2005. 
22 Martin Roller. Poc sets, median algebras and group actions. preprint, University of Southampton, 1998.

23 Michah Sageev. Ends of group pairs and non-positively curved cube complexes. Proc. London Math. Soc., 71:585-617, 1995.

24 Micha Sharir. On shortest paths amidst convex polyhedra. SIAM J. Comput., 16:561-572, 1987. 